\title{
Dielectric, NMR and x-ray diffraction study of pseudo-one-dimensional Cs1- $\mathrm{x}(\mathrm{NH} 4) \mathrm{xH} 2 \mathrm{PO} 4$
}

\section{Authors: S. Maschia, S. Lanceros-Mendez, A. Zidansek, V. Hugo Schmidt, and R. Larsen}

This is an Accepted Manuscript of an article published in Ferroelectrics in 1999, available online: https://www.tandfonline.com/10.1080/00150199908230297.

S. Meschia, S. Lanceros-Mendez, A. Zidansek, V.H. Schmidt, and R. Larsen, "Dielectric, NMR and x-ray diffraction study of pseudo-one-dimensional Cs1-x( $\mathrm{NH} 4) \times \mathrm{H} 2 \mathrm{PO} 4$," Ferroelectrics 226, 159-167 (1999). doi: 10.1080/00150199908230297. 


\title{
DIELECTRIC, NMR AND X-RAY DIFFRACTION STUDY OF PSEUDO-ONE-DIMENSIONAL $\mathrm{Cs}_{1-x}\left(\mathrm{NH}_{4}\right)_{x} \mathrm{H}_{2} \mathrm{PO}_{4}$
}

\author{
S. MESCHIA ${ }^{a}$, S. LANCEROS-MÉNDEZ ${ }^{\text {a }}$, A. ZIDANŠEK ${ }^{a}$, \\ V. H. SCHMIDT ${ }^{\mathrm{a}, *}$ and R. LARSEN ${ }^{\mathrm{b}}$ \\ ${ }^{a}$ Dept. of Physics, Montana State University, Bozeman, MT 59717, USA; \\ ${ }^{b}$ Dept. of Chemistry, Montana State University, Bozeman, MT 59717, USA
}

(Received in final form 17 July 1998)

Mixed crystals $\mathrm{Cs}_{1-x}\left(\mathrm{NH}_{4}\right)_{x} \mathrm{H}_{2} \mathrm{PO}_{4}$ of the ferroelectric $\mathrm{CsH}_{2} \mathrm{PO}_{4}(\mathrm{CDP})$ and the antiferroelectric $\mathrm{NH}_{4} \mathrm{H}_{2} \mathrm{PO}_{4}$ were grown with $x=0.2$ (CADP0.2) in solution. The structural properties of the crystal were analyzed by means of X-ray diffraction. Dielectric measurements at several temperatures and frequencies have been performed along the three crystallographic axes in this sample and also in the fully deuterated CADP0.2 sample (DCADP0.2). Dielectric and NMR experiments in a powdered sample were also performed. The shift of the transition temperature as a function of $x$ and deuteration, and the changes in the properties of the different phases together with, the thermally-activated conductivity found in the paraelectric phase will be discussed and related with the several relaxation mechanisms measured in the NMR experiments.

Keywords: Phase transitions; mixed crystals; CADP

\section{INTRODUCTION}

Crystals that contain mixtures of ferroelectrics and antiferroelectrics have interesting properties due to the frustration resulting from the opposing orderings. Many questions remain about the spatial and temporal behavior of dipolar domains and coexistence in such crystals.

$\mathrm{CsH}_{2} \mathrm{PO}_{4}(\mathrm{CDP})$ is known to have one-dimensional ferroelectric order below $T=159 \mathrm{~K}$ and a superionic transition at $T=504 \mathrm{~K}$ on heating, ${ }^{[1]}$

\footnotetext{
* Corresponding author.
} 
whereas $\mathrm{NH}_{4} \mathrm{H}_{2} \mathrm{PO}_{4}$ (ADP) is antiferroelectric below $T=148 \mathrm{~K}^{[2]}$ In addition, their paraelectric phases have different structures; $\mathrm{CDP}$ is monoclinic with the space group $\mathrm{P} 2 / \mathrm{m}$ and $\mathrm{ADP}$ is tetragonal with the space group $\bar{I} \overline{4} 2 d$.

In this work we present $\mathrm{X}$-ray, dielectric and NMR results in undeuterated, deuterated and powdered $\mathrm{Cs}_{1-x}\left(\mathrm{NH}_{4}\right)_{x} \mathrm{H}_{2} \mathrm{PO}_{4}$ crystals with $x=0.2$ in solution.

\section{EXPERIMENTAL METHOD}

The crystals were grown by controlled evaporation from aqueous solution of $20 \%$ molar concentration of ADP and $80 \%$ of CDP.

In order to perform the $\mathrm{X}$-ray transmission experiments small semispherical samples $\left(\sim 1 \mathrm{~mm}^{3}\right)$ were prepared in order to reduce the effect of the extinction coefficients on the transmission spectra. From this experiment the crystal quality and crystal structure, including bond lengths and angles, were determined. An upper limit for $x$ was also set by this method. The value of $x$ was finally determined after a chemical analysis by the Kjeldahl method. ${ }^{[3]}$

For the dielectric measurements, samples in the shape of thin plates $(<1 \mathrm{~mm})$ were cut perpendicular to the three crystallographic axes, optically polished and silver painted. The temperature $(25 \mathrm{~K}-320 \mathrm{~K})$ and frequency $(10 \mathrm{~Hz}-1 \mathrm{MHz})$ dependences of $\varepsilon^{\prime}$ and $\tan \delta\left(=\varepsilon^{\prime \prime} / \varepsilon^{\prime}\right)$ were measured with two different impedance meters (Wayne-Kerr 6425 and Quad Tech 7600).

For the NMR measurements, in order to maximize the fill factor of the sample coil, several crystals were pulverized and desiccated for several days. The variation of the spin-lattice relaxation time as a function of temperature was measured. For each $T_{1}$ data point the temperature was maintained to within a tenth of a degree over a period of days by means of a Lake Shore DR91C Temperature Controller. The error in the value of $T_{1}$, as estimated from the fits and the thermal drift during the experiment, could be as much as $10 \%$.

\section{RESULTS}

\section{X-ray and Chemical Analysis}

$\mathrm{X}$-ray diffraction experiments were used to determine ammonium concentration, crystal quality, and crystal structure, including bond lengths and 
angles. Crystal quality, a concern for mixed crystals, was excellent. The lattice structure and the space group of CADP0.2 was found to be identical to that of pure CDP at room temperature, monoclinic with the space group $\mathrm{P} 21 / \mathrm{m}$. The measured lattice parameters were: $a=7.893(67) \AA$, $b=6.383(46) \AA, c=4.876(13) \AA$, and $\beta=107.56(06) \mathrm{deg}$. There were small variations between the parameters of different crystals of the same concentration but, in general, the values were very close to those in the pure compound, ${ }^{[4]}$ with the most significant variation being a small increase in length $(\sim 0.02 \AA)$ along the ferroelectric $b$-axis. It was also observed from $\mathrm{x}$-ray intensity analysis that a much smaller concentration of ammonium was present in the crystals than in the solution from which they grown: the crystals were found to have $x=0.05 \pm 0.05$. Further chemical analysis of the crystals showed the ammonium concentration to be $0.047 \pm 0.002$.

\section{Dielectric Measurements}

The anomalies in $\varepsilon_{b}^{\prime}(T)$ and $\varepsilon_{b}^{\prime \prime}(T)$ are similar to those observed in the pure compound (Fig. 1). The measured transition temperature $\left(T_{c}=159.3 \mathrm{~K}\right)$ and the shape and frequency dependence of the anomalies show small changes caused by variations of ammonium concentration. The transition temperature is shifted $\sim 3 \mathrm{~K}$ to higher temperatures. The fitting parameters of the quasi-1D-Ising model ${ }^{[5-8]}$ suffer significant variations with the increase of concentration, while at the same time the dielectric permittivity along the $c$ - and $a$-axes clearly show anomalies around the transition temperature into the ferroelectric phase (Fig. 2). These anomalies do not exist in the pure compound.

The $\varepsilon_{b}^{\prime}(T)$ curves in both the undeuterated and deuterated crystals could be well fitted with the quasi-1D-Ising model. The experimental $\varepsilon_{b}^{\prime}(T)$-curves were fitted in the temperature regions $T>T_{c}+(\approx 5 \mathrm{~K})$ according to the equation

$$
\varepsilon^{\prime}(T)=\varepsilon_{\infty}+\left[\left(C /\left(T-T_{c}\right)\right]\left[\exp \left(-2 J_{\|} / T\right)-J_{\perp} / T\right]^{-1} ;\right.
$$

where the intrachain $J_{\|}$and the interchain $J_{\perp}$ interaction constants are given in temperature units. The values obtained for the adjustable parameters are summarized in Table I, and a typical fit is shown in Figure 3.

The effect of increasing ammonium impurities is a decrease in the interactions along the chains parallel to the ferroelectric axis and an increase of the interactions between the chains, perpendicular to that axis. ${ }^{[5-8]}$ This means an increase in the isotropy of the crystal, becoming more three- 

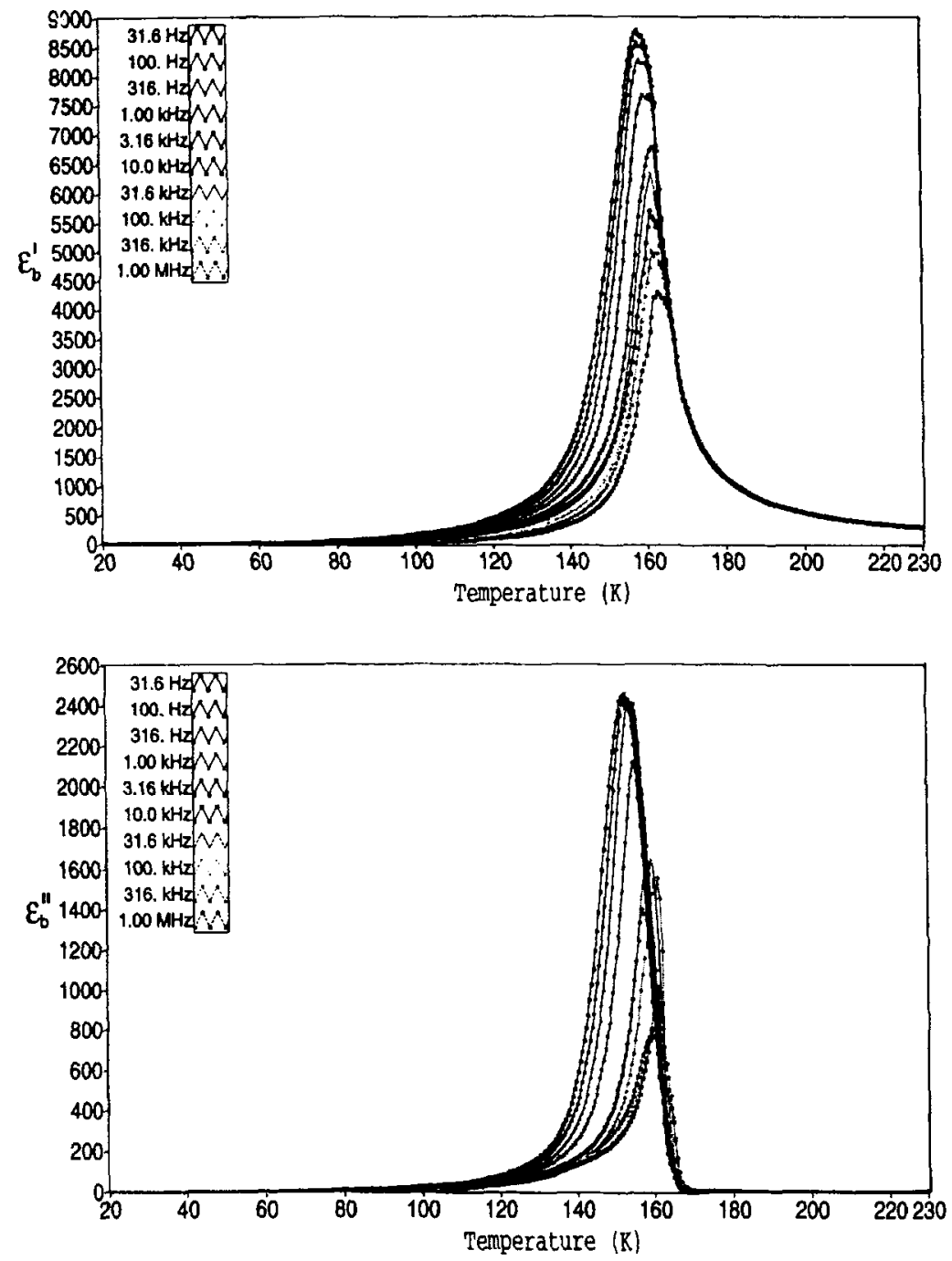

FIGURE $1 \quad \varepsilon_{b}^{\prime}(T)$ and $\varepsilon_{b}^{\prime \prime}(T)$ for CADP0.2 at several frequencies.

dimensional than pure CDP. This fact was confirmed by performing dielectric experiments along the $a$ - and $c$-axis: in both cases dielectric anomalies were found that do not exist in pure CDP. The anomaly along the $a$-axis is about 10 times lower than the one along the $b$-axis (Fig. 2) and the anomaly along the $c$-axis is about 100 times lower.

A striking feature observed in the dielectric measurements is the apparently thermally activated conductivity within the paraelectric phase 

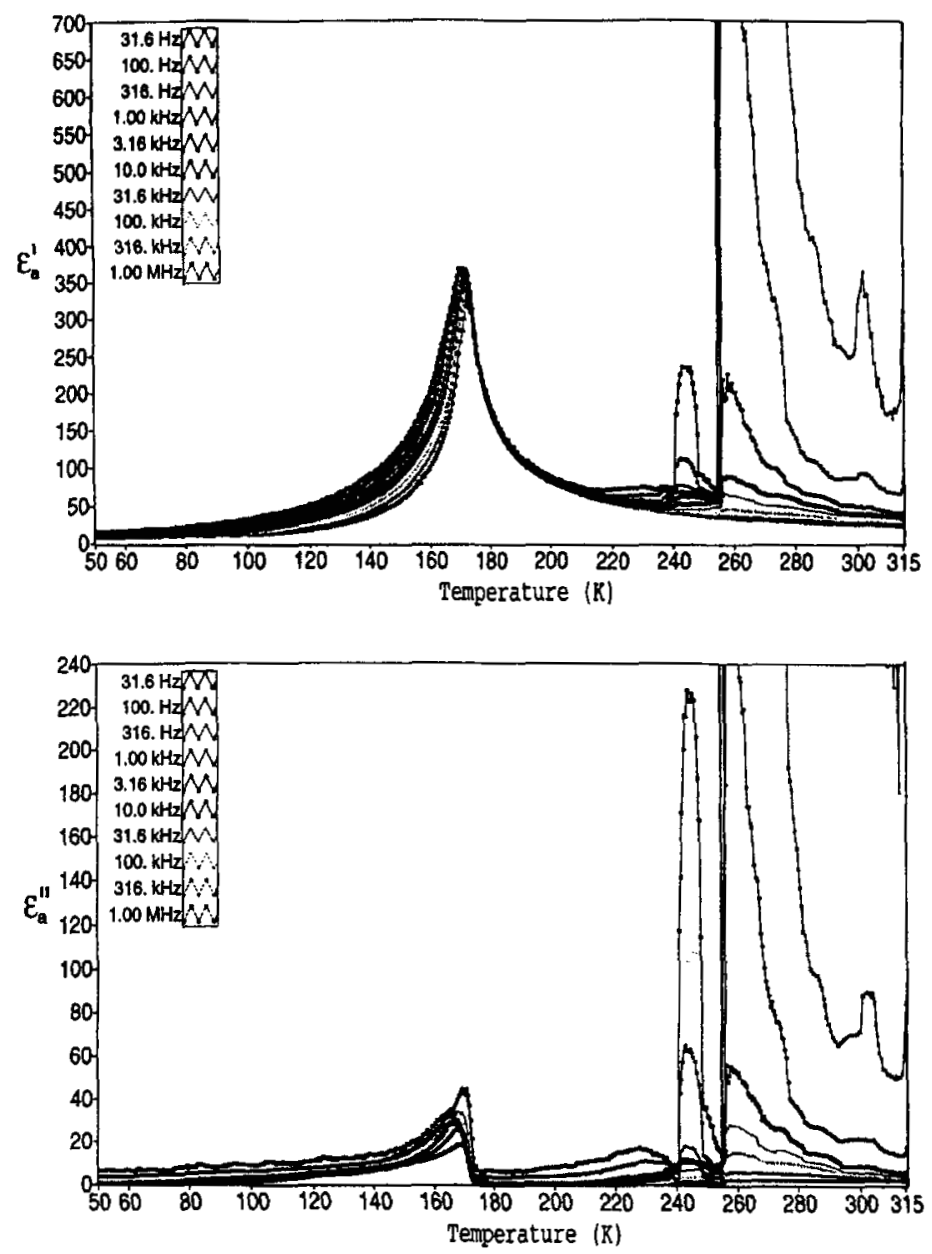

FIGURE $2 \varepsilon_{a}^{\prime}(T)$ and $\varepsilon_{a}^{\prime \prime}(T)$ for CADP0.2 at several frequencies.

along the $a$-and $c$-axes. Similar dielectric anomalies occur in many other mixed crystals isomorphs of KDP containing ammonium, as well as in pure ADP. Comparison with our results from NMR suggests that the large anomaly is related to conductivity arising from the protons or deuterons. Cycling the experiments several times demonstrates that the conductivity effects present a very large $(\sim 40 \mathrm{~K})$ and anomalous thermal hysteresis that depends on the thermal history of the crystal: in the heating runs the conductivity starts at lower temperatures than it stops in the cooling runs. The anomalies due to this conductivity were also observed in the deuterated and powdered samples. 
TABLE I Fit parameters of the quasi-1D-Ising model for CADP0.2 and DCADP0.2

\begin{tabular}{lcccc}
\hline Compound & $C$ & $J_{\perp} / K$ & $J_{\|} / K$ & $\varepsilon_{\infty}$ \\
\hline CADP-0.2 & 12860 & 14 & 156 & 48 \\
DCADP-0.2 & 5766 & 17 & 365 & 34 \\
\hline
\end{tabular}

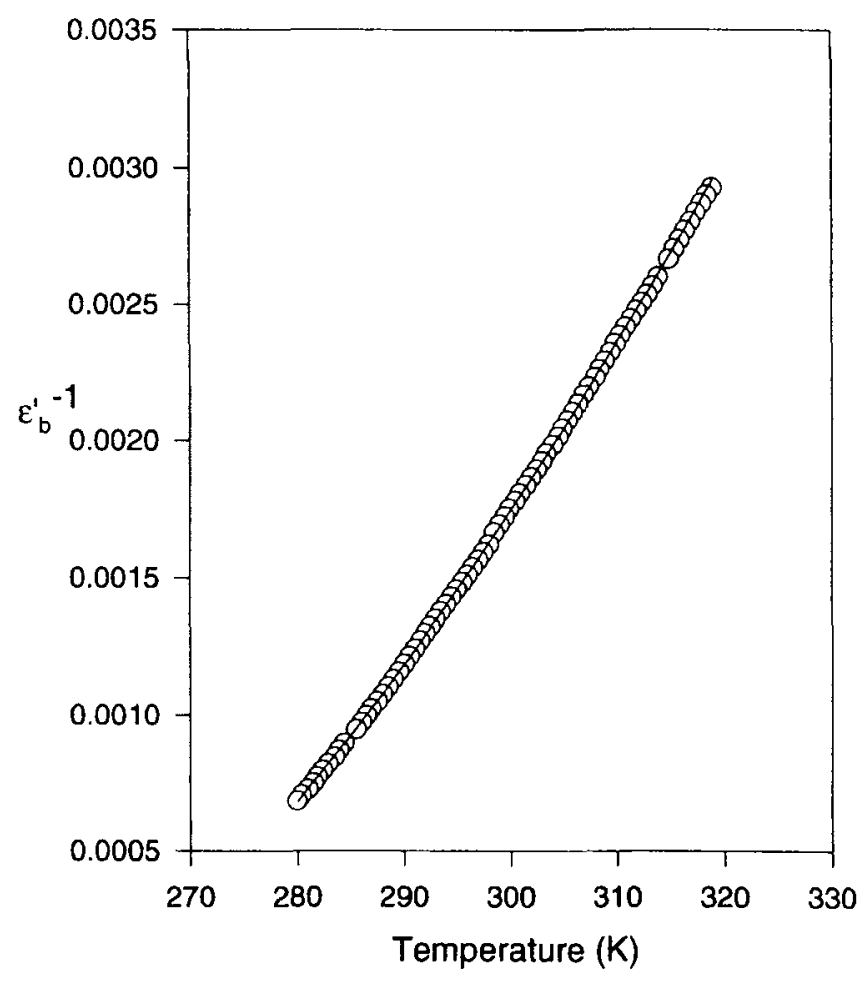

FIGURE 3 Fit of the quasi-1D-ising model for DCADP0.2 at $1 \mathrm{kHz}$.

The effect of deuteration in CADP0.2 is similar to that in pure CDP: the transition temperature is shifted from $159.3 \mathrm{~K}$ to $276 \mathrm{~K}$, compared to a shift from $156 \mathrm{~K}$ to $268.3 \mathrm{~K}$ in the pure compound. ${ }^{[5]}$ The changes in shape, as represented by the values of the adjustable parameter of the quasi-1D-Ising model (Tab. I), the anisotropy and the frequency dispersion are as described for the undeuterated sample. The temperature and shape of the conductivity effects remain almost independent of the amount of deuteration.

Dramatic differences are observed between dielectric responses of crystalline and powdered CADP0.2; the characteristic features of the 
powdered sample are the disappearance of the high ferroelectric anomaly and the appearance of a small rounded maximum at a temperature $4 \mathrm{~K}$ higher, and an abrupt and frequency dependent drop in $\varepsilon^{\prime}(T)$ accompanied by an anomaly in $\varepsilon^{\prime \prime}(T)$ (Fig. 4). The behavior of the second feature strongly resembles the typical behavior of dipole glasses, which, together with the anomaly near the transition temperature into the ferroelectric phase, prompted some authors to conclude that the related compound CADA exhibited coexistence of ordered (ferro- or antiferroelectric) and glassy phases. ${ }^{[9]}$
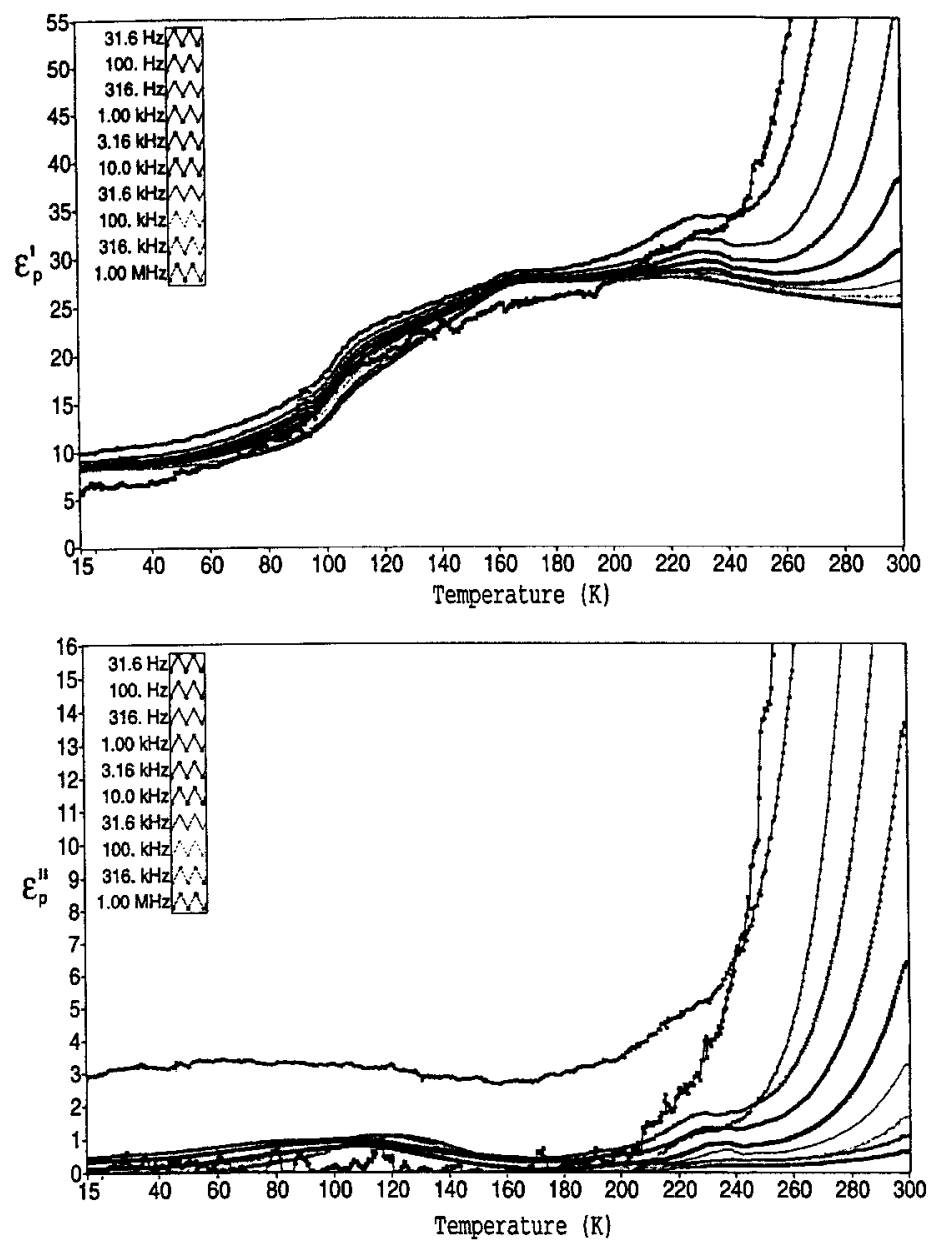

FIGURE $4 \varepsilon_{p}^{\prime}(T)$ and $\varepsilon_{p}^{\prime \prime}(T)$ for powdered CADP0.2 at several frequencies. 


\section{Nuclear Magnetic Resonance Measurements}

The primary mechanism for the ${ }^{31} \mathrm{P} T_{1}$ relaxation in our sample is dipolar coupling between hydrogen $(\mathrm{H})$ and phosphorus $(\mathrm{P})$ nuclei in the lattice. $\mathrm{H}$ is the more mobile nuclear species, implying that the relaxation time $\tau$ is characteristic of a specific motion of the $\mathrm{H}$-atom. Assuming liquid-like motion of the hydrogens, for a static $\mathrm{NMR}$ field $\mathrm{H}_{0}$ giving $28 \mathrm{MHz}$ resonant frequency for ${ }^{31} \mathrm{P}$ and nuclear separation $\mathrm{r}$, yields $T_{\mathrm{l}(\min )}=\left(8.89 \times 10^{57}\right.$ $\left.\mathrm{m}^{-6} \mathrm{~s}\right) \mathrm{r}^{6}$.

The molecular radius of the ammonium ions is considerable smaller than that of the Cs ions for which they substitute. Thus for small concentrations of ammonium the molecular structure will be that of CDP with the ammonium ions free to move within the larger $\mathrm{Cs}^{+}$cation space. The distance of closest approach for the $\mathrm{H}$ ions in the ammonium groups will be $2.2 \times 10^{-10} \mathrm{~m}$ to fit easily in between. This distance yields a $T_{1}$ value of $1.0 \mathrm{~s}$ at the minimu.

We calculated the distance of closest approach of acid protons to phosphorus, obtaining $2.63 \times 10^{-10} \mathrm{~m}$. This yields a $T_{1}$ minimum of $2.9 \mathrm{~s}$.

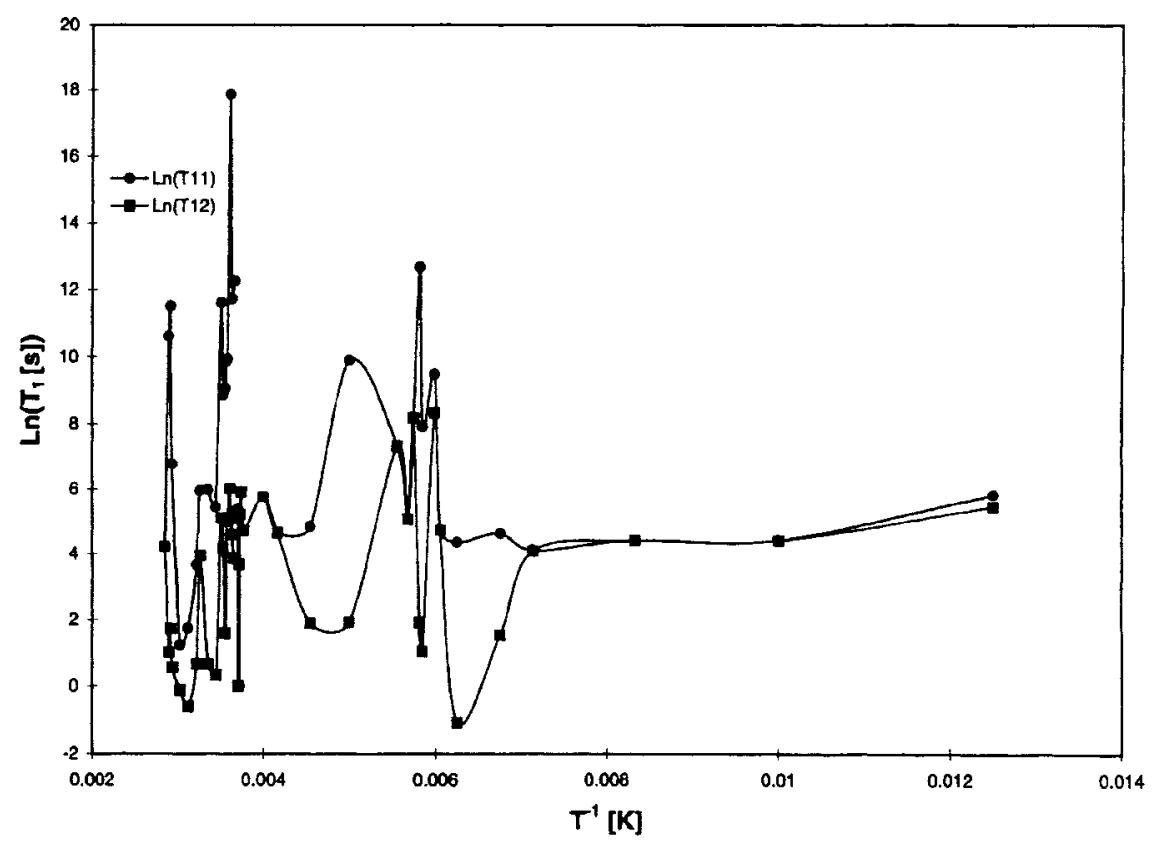

FIGURE 5 Natural logarithm of $T_{11}$ and $T_{12}$ versus inverse temperature. $T_{11}$ and $T_{12}$ correspond to the $T_{1}$ values of the two different processes. 
We tentatively associate the minimum in the graph of $\ln \left(T_{1}\right)$ versus temperature (Fig. 5) at $162 \mathrm{~K}$ with ammonium hindered rotation and the minima at $328 \mathrm{~K}$ with $\mathrm{H}$ interbond motion. We expect that the effect of intrabond motion is too small to detect by ${ }^{31} \mathrm{P}$ relaxation. By minimizing $T_{1}$ as a function of temperature, the frequency $f$ of the processes at these minima is determined to be $38.9 \mathrm{MHz}$.

Assuming an activated process with activation energy $E_{A}$, the relaxation times $\tau$ of the motions at arbitrary temperatures can be calculated from $\tau=\tau_{0} \exp \left(E_{A} / k T\right)$. Setting the energy of motion equal to the thermal energy at the minimum, we obtain $E_{A}=k T \ln (k T / h f)$, where $k$ is the Boltzmann's constant and $h$ is Planck's constant. This equation yields values of $0.35 \mathrm{eV}$ at $328 \mathrm{~K}$, and $0.16 \mathrm{eV}$ at $162 \mathrm{~K}$. The experimental activation energies $E_{A}$ as determined from the slope of the $\ln \left(T_{1}\right)$ versus reciprocal temperature curves near the minima systematically diverge from these results with increasing temperature. We obtain $E_{A}=0.46 \mathrm{eV}$ for the acid relaxation at $328 \mathrm{~K}$, and $E_{A}=0.19 \mathrm{eV}$ for the ammonium relaxation at $162 \mathrm{~K}$. Since intrabond motion has little effect on $T_{1}$, then the process near $328 \mathrm{~K}$ most probably corresponds to an actual proton mobility between lattice sites.

\section{Acknowledgements}

Work supported from NSF grant DMR-9520251. S. L. M. gratefully thanks the Basque Government for support under grant BFI96.041.

\section{References}

[1] Baranov, A. I., Khiznichenko, V. P. and Shuvalov, L. A. (1989). Ferroelectrics, 100, 136.

[2] Eichele, K. and Wasylishen, R. E. (1994). J. Phys. Chem., 98, 3108.

[3] Uesu, Y. and Kobayashi, J. (1976). Phys. Status Solidi (a), 34, 475.

[4] Simone, A. H., Simone, E. H. and Cresman III, C. P. (1997). J. Sci. Food Ag., 73, 39.

[5] Deguchi, K., Okaue, E. and Nakamura, E. (1982). J. Phys. Soc. Jpn., 51, 3569.

[6] Blinc, R., Žekš, B., Levstik, A., Filipic, C., Slak, J., Burgar, M., Zupančič, I., Shuvalov, L. A. and Baranov, A. I. (1979). Phys. Rev. Lett., 43, 231.

[7] Kanda, E., Yoshizawa, M., Yamakani, T. and Fujimura, T. (1982). J. Phys. C, $15,6823$.

[8] Imai, K. (1983). J. Phys. Soc. Jpn., 52, 3960.

[9] Song, T. K., Moon, S. E., Noh, K. H., Kwun, S. I., Shin, H. K. and Kim, J. J. (1994). Phys. Rev. $B, 50,6637$. 